\title{
Delivering bad news: patient's perspective and opinions
}

This article was published in the following Dove Press journal:

Patient Preference and Adherence

\section{Krzysztof Sobczak \\ Katarzyna Leoniuk \\ Agata Janaszczyk}

Department of Social Medicine and Social Pathology, Medical University of Gdansk, Gdansk, Poland
Correspondence: Krzysztof Sobczak Department of Social Medicine and Social Pathology, Medical University of Gdansk, Tuwima I5 Str, 80-2I0 Gdansk, Poland Tel/fax +48 58349 I55 I

Email krzysztof.sobczak@gumed.edu.pl
Purpose: The aim of our research was to gain knowledge about patients' opinions, experiences, and preferences with regard to the way the news is being delivered to them.

Materials and methods: Detailed research was carried out on a group of 314 patients using the CAWI (Computer-Assisted Web Interview) technique. Adult responders who had earlier received bad news were questioned about their opinion about the way the doctor acted while delivering bad news and how he did it.

Results: Patients, who define the following aspects of their visit as negative/lacking: 1) doctor's behavior in the moment of delivering bad news, 2) amount of time devoted to the visit, 3) lack of doctor's attention, 4) usage of medical terminology, 5) doctor's honesty, 6) emotional and cognitive support from the doctor, more often tend to change the doctor in charge of their therapy or decide to cease the medical treatment.

Conclusion: Doctors' behavior and the way they deliver news to patients are key elements that strongly influence patients' future therapy. It makes an impact on patient's decision whether to continue or cease the treatment. In the first case, it also leads the patient to choose to continue the treatment under the guidance of the same specialist or to find another one. The data that we acquired and that we will discuss below will form the basis for editing a communication protocol concerning delivering bad news. It is necessary to create such a protocol in order to improve the quality of communication with patients, especially as regards delivering bad news to them.

Keywords: diagnosis, truth disclosure, doctor-patient relationship

\section{Introduction}

Delivering bad news $(\mathrm{DBN})$ is one of the hardest challenges that a doctor faces in their medical practice. ${ }^{1}$ An inappropriate way of communicating with the patient can have a huge impact on the way they perceive their disease, it can also influence whether they quit or continue the medical treatment. Many studies have shown that there is a direct relation between the doctors' communication skills and the therapeutic results. ${ }^{2}$ Approaching the patient with empathy, using accurate language, and engaging the patient in the decision-making process - these are the basic rules that build a proper relationship between the doctor and the patient. Medical communication definitely has a therapeutic dimension. It determines the growth of patient's trust toward the doctor; ${ }^{3}$ it also influences the medical treatment's results ${ }^{2}$ as well as patient's satisfaction with medical services. ${ }^{4,5}$ If we look at the benefits of the proper medical communication from the doctor's perspective, we can see that it prevents work burnout ${ }^{6}$ and raises the level of self-efficacy. ${ }^{7}$

Our earlier studies indicated that there are two main factors that escalate doctors' stress level in the process of DBN. ${ }^{8,9}$ The first of these factors is the education gap in terms of soft skills, which is present in the process of education of medical university 
students in Poland. The second factor is paternalistic viewpoint among doctors. The results of our studies and our personal experience related to the education of future physicians have led us to investigate this problem from the patients' point of view. We would like our research to contribute to a more complete identification of the training and educational needs of physicians. Thanks to such assessment, we will be able to develop and implement more relevant teaching content into the education of physicians.

Our research aimed at studying perception and preferences of patients who received bad news. We wanted to know the patients' view on the doctor's behavior during the process of DBN. In our study, we assumed that "bad news" is any form of diagnosis related to permanent or relatively permanent changes in the organism that requires continuous or long-term medical treatment or a therapy focused on pain management. If we view bad news from this perspective, we see that it encompasses somatic diseases (eg, diabetes, coronary disease, allergies, different types of tumors) as well as mental illnesses, genetic diseases, and incurable fatal diseases.

\section{Materials and methods}

The research was carried out in the period between February and October 2017. The data were collected using the CAWI (Computer-Assisted Web Interview) technique. We used a specially designed e-questionnaire as our research tool. In the process of creating the questionnaire, we took into account the experience gathered by us in previous research projects concerning the physician-patient relationship. Additionally, we conducted methodological consultations of the research tool that aimed at assessing the correctness and unambiguity of the formulated questions. Patients were asked 16 closed questions and three semiopen questions. The questionnaire consists of eight closed questions, two semiopen questions, and one open question - concerning the disease unit. The study group $(\mathrm{N}=314)$ was fully inclusive, and the participants were chosen at random. Participation in the study was anonymous and voluntary. Information about the study was delivered to patients via national independent patients' organizations and associations as well as via electronic media. The survey's instructions informed the participants that, by completing the survey, they gave consent to take part in the research and the data obtained would be processed anonymously in aggregate statistical summaries.

In the final analysis, only fully completed questionnaires were taken into account. The request to publish information about the survey together with a link to the e-questionnaire was addressed to 98 foundations and associations operating in Poland. The cooperation was undertaken by 19 organizations. Moreover, information about the survey was published on 30 Internet forums administered by health-related portals. For the purposes of statistical analysis, SPSS v.26.0 software was used. Opinions and assessments of the patients were correlated with the sociodemographic variables (age, gender, marital status, education, and place of residence), health variables (type of disease), and medical variables (DBN doctor's specialty, place of contact with the doctor, and type of the payer who covers the cost of the visit). We have also used Pearson's chisquared test for analyzing the correlation between discontinuous variables and the statistic heterogeneity of the groups. We have assumed the difference for $P<0.05$ as statistically important.

\section{Results}

Due to the fact that an e-questionnaire was used, we have observed a typical overrepresentation of women $-79 \%$ of the study group $(\mathrm{N}=248)$, young people $(<30)$, and highly educated (59\%). In most cases, the patients received bad news in the hospital. One of the study group's features was a wide diversity of diseases. For the purpose of classifying disease entities, ICD-10 was used. Most of the respondents were suffering from cancer (38\%), only one out of four respondents was suffering from nervous system illnesses (19\%), and one out of ten respondents ( $9 \%$ ) was suffering from disorders of pancreatic internal secretion. Bad news was delivered to patients by doctors representing 15 different specialities (Table 1). Patients' decision concerning their further contact with the doctor who delivered bad news to them also turned out to be an important variable in our study. Precisely, $49 \%$ of the patients decided to continue their medical treatment under the guidance of the doctor who delivered bad news, $45.5 \%$ decided to change the doctor managing their therapy, and $5.5 \%$ withdrew from continuing their therapy.

\section{Evaluation of the way bad news was being delivered}

The Code of Medical Ethics currently prevailing in Poland (article 17) imposes upon the doctor the responsibility of DBN to the patient with "with tact and care." Almost half of the patients (47\%) said that doctors delivered bad news in a proper way. One out of five patients $(20 \%)$ is unable to assess the doctor's behavior and one out of three patients (33\%) stated that the doctors have violated the abovementioned ethical obligation and were tactless and/or acted without proper care while DBN. 
Table I Characteristics of respondents $(\mathrm{N}=3 \mid 4)$

\begin{tabular}{|c|c|c|}
\hline & $\mathbf{N}$ & $\%$ \\
\hline \multicolumn{3}{|l|}{ Gender } \\
\hline Female & 248 & 79 \\
\hline Male & 66 & 21 \\
\hline \multicolumn{3}{|l|}{ Education } \\
\hline Primary education & 4 & 1.5 \\
\hline Lower-secondary education & 4 & 1.5 \\
\hline Vocational education & 15 & 5 \\
\hline Secondary education & 105 & 33 \\
\hline Higher education & 186 & 59 \\
\hline \multicolumn{3}{|l|}{ Place where bad news was delivered } \\
\hline Public clinic & 47 & 15 \\
\hline Public polyclinic & 45 & 14.5 \\
\hline Private polyclinic & 20 & 6.5 \\
\hline Private clinic & 8 & 2.5 \\
\hline Private doctor's office & 29 & 9 \\
\hline Hospital & 165 & 52.5 \\
\hline \multicolumn{3}{|l|}{ Type of disease } \\
\hline Tumors & 119 & 38 \\
\hline Nervous system diseases & 61 & 19 \\
\hline Disorders of pancreatic internal secretion & 29 & 9 \\
\hline Blood disorders and cardiovascular diseases & 26 & 8 \\
\hline Musculoskeletal diseases & 18 & 6 \\
\hline Genitourinary system's diseases & 14 & 5 \\
\hline Mental and behavioral disorders & 9 & 3 \\
\hline Skin diseases & 9 & 3 \\
\hline Eye diseases & 8 & 2.5 \\
\hline Other & 21 & 6.5 \\
\hline \multicolumn{3}{|l|}{ Marital status } \\
\hline Single & 111 & 35.5 \\
\hline Married & 167 & 53 \\
\hline Widow/widower & 17 & 5.5 \\
\hline Divorced & 19 & 6 \\
\hline \multicolumn{3}{|l|}{ Age } \\
\hline $18-30$ years & 78 & 25 \\
\hline $31-40$ years & 84 & 27 \\
\hline $4 I-50$ years & 65 & 21 \\
\hline $5 \mathrm{I}-60$ years & 44 & 14 \\
\hline 61 years and more & 43 & 13 \\
\hline \multicolumn{3}{|l|}{ Visit payer } \\
\hline National Health Fund & 254 & 81 \\
\hline Insurance company & 5 & 1.5 \\
\hline Own resources & 46 & 14.5 \\
\hline I do not remember & 9 & 3 \\
\hline \multicolumn{3}{|l|}{ Medical specialization } \\
\hline Neurologist & 54 & 17 \\
\hline Hematologist & 41 & 13 \\
\hline
\end{tabular}

Table I (Continued)

\begin{tabular}{|l|l|l|}
\hline & N & $\%$ \\
\hline Oncologist & 37 & 12 \\
\hline Surgeon & 29 & 9 \\
\hline Gynecologist & 25 & 8 \\
\hline Endocrinologist & 21 & 7 \\
\hline General physician & 21 & 7 \\
\hline Rheumatologist & 10 & 3 \\
\hline Cardiologist & 10 & 3 \\
\hline Other: psychiatrist, etc & 66 & 21 \\
\hline
\end{tabular}

We have noticed that $64 \%$ of patients who positively evaluated the doctor's behavior in DBN (answers: "definitely yes" and "I guess so") continued their medical therapy with this doctor. On the contrary, $50 \%$ of the patients who decided to change the specialist or totally resign from further therapy negatively evaluated the doctor's behavior $\left(\chi^{2}=46.405\right.$; $d f=2 ; P<0.01)$.

\section{The amount of time devoted to the patient's visit}

Providing the patient with the sense of an adequate amount of time for the visit is an important element in DBN. Fortytwo percent of respondents stated that doctors did not devote enough time for DBN, whereas $37 \%$ of respondents stated the opposite. Exactly $21 \%$ of patients were unable to evaluate this element of visit.

Similar to the evaluation of the way in which bad news was delivered also in studying the time factor, we have obtained substantial statistic correlations. Half of the patients (52\%) who decided to continue their treatment under the guidance of the specialist DBN also gave a positive evaluation of the time factor saying that the doctor has devoted an adequate amount of time for DBN. One out of four patients (25\%) gave a negative evaluation of the time factor. Over half of the $(59.5 \%)$ patients who decided to continue their medical therapy under the guidance of another doctor or who decided to withdraw from therapy gave a strongly negative evaluation of the time factor of the visit $\left(\chi^{2}=41.773\right.$; $d f=2$; $P<0.01)$.

\section{Evaluation of the completeness of received information}

In order to analyze deep and specific aspects of DBN, we asked the patients whether in their opinion they were given comprehensive explanation about the causes and effects of the diagnosis (Table 2). In the view of respondents, such 
Table 2 Evaluation of the completeness of information related to bad news $(N=3 \mid 4)$

\begin{tabular}{|l|l|l|l|l|l|l|}
\hline \multirow{2}{*}{$\begin{array}{l}\text { Did the doctor inform the } \\
\text { patient about }\end{array}$} & \multicolumn{2}{|l|}{$\begin{array}{l}\text { The doctor informed } \\
\text { the patient in a fully } \\
\text { comprehensive way }\end{array}$} & $\begin{array}{l}\text { The doctor informed } \\
\text { the patient in a partially } \\
\text { comprehensive way }\end{array}$ & $\begin{array}{l}\text { The doctor did not inform } \\
\text { the patient }\end{array}$ \\
\cline { 2 - 7 } & $\mathbf{N}$ & $\%$ & $\mathbf{N}$ & $\%$ & $\mathbf{N}$ & $\%$ \\
\hline Causes of the disease & 78 & 25 & 109 & 35 & 127 & 40 \\
\hline Prognosis & 107 & 34 & 146 & 46 & 61 & 19 \\
\hline Further treatment & 118 & 37 & 106 & 33.5 & 90 & 28.5 \\
\hline Different methods of treatment & 85 & 27 & 87 & 28 & 142 & 45 \\
\hline Consequences of the lack of treatment & 95 & 30 & 71 & 27 & 148 & 47 \\
\hline $\begin{array}{l}\text { Recommendations related to everyday } \\
\text { conduct (eg, diet, physical activities) }\end{array}$ & 83 & 26 & 91 & 29 & 140 & 45 \\
\hline
\end{tabular}

aspects as the prognosis and details about further diagnostic tests are among the details that the doctors most often inform the patients about. Most of the patients stated that they received too little information about the variety of treatment methods and about the causes of the illness.

The patients evaluating the completeness of received information were divided into two groups. The first group comprised patients who declared that the information they received from the doctor was fully comprehensive or partially comprehensive. The second group comprised patients who reported lack of information. We correlated the evaluation delivered by both groups with the sociodemographic variables. By doing so, we received statistically important correlations. We observed that acquiring detailed information from the doctor (such as prognosis, consequences of quitting further medical therapy, and indications concerning everyday procedures that the patient is advised to take) depends on the gender of the patient. In all the cases, it was more often men (91\%) than women (78\%) confirming that they have received fully comprehensive or partially comprehensive information $\left(\chi^{2}=5.703 ; d f=1 ; P=0.017\right)$. As regards fully comprehensive or partially comprehensive information about potential effects of quitting the medical treatment, it was delivered to $65 \%$ of male patients and half of female patients $(50 \%)$ for values $\left(\chi^{2}=5.062 ; d f=1 ; P=0.024\right)$. Furthermore, information (fully comprehensive or partially comprehensive) about the medical recommendations concerning patient's lifestyle was delivered to $70 \%$ of men and $52 \%$ of women for values $\left(\chi^{2}=6.900 ; d f=1 ; P=0.009\right)$.

\section{Quality of communication with a doctor}

In our study, we also asked our respondents about the quality markers of patient-doctor communication (Table 3). Twenty-five percent of the patients stated that during DBN the doctor was using medical terms that the patients could not understand. Only two out of ten patients said that the doctor encouraged them to ask questions about the diagnosis. Only $12 \%$ of the patients stated that the doctors supplied them with additional sources of information about the disease that the patients were diagnosed with.

Doctors' usage of incomprehensible medical terminology was more often reported by patients using the national health service $(30 \%)$ than patients who use private health care $(8.5 \%$; statistics: $\left.\chi^{2}=11.877 ; d f=2 ; P<0.01\right)$. On the contrary, patients also reported incidents of doctors downplaying the diagnosis. This situation was reported by patients under 40 years of age (27\%) much more often than by patients at 41 years and older (16\%; statistics: $\left.\chi^{2}=10.419 ; d f=2 ; P<0.01\right)$.

Again, the evaluation of doctor's behavior during DBN correlated with patients' decision to continue or quit the

Table 3 Evaluation of the doctor's behavior during DBN $(\mathrm{N}=3 \mid 4)$

\begin{tabular}{|c|c|c|c|c|c|c|}
\hline \multirow[t]{2}{*}{ Did the doctor use any of the following behavior patterns while DBN } & \multicolumn{2}{|c|}{ Yes } & \multicolumn{2}{|l|}{ No } & \multicolumn{2}{|c|}{ I do not remember } \\
\hline & $\mathbf{N}$ & $\%$ & $\mathbf{N}$ & $\%$ & $\mathbf{N}$ & $\%$ \\
\hline Used incomprehensible medical terminology & 81 & 26 & 187 & 59.5 & 46 & 14.5 \\
\hline Downplayed the problem & 68 & 22 & 221 & 70 & 25 & 8 \\
\hline Encouraged the patient to ask questions & 73 & 23 & 196 & 63 & 45 & 14 \\
\hline Encouraged the patient to individually deepen his/her knowledge about the disease & 61 & 19 & 219 & 70 & 34 & 11 \\
\hline Informed the patient about the sources of additional information about the disease & 38 & 12 & 246 & 78 & 30 & 10 \\
\hline
\end{tabular}

Abbreviation: DBN, delivering bad news. 
Table 4 Patient's feelings during DBN $(\mathrm{N}=3 \mid 4)$

\begin{tabular}{|l|l|l|l|l|l|l|}
\hline While receiving bad news I felt that the doctor & \multicolumn{2}{l|}{ Yes } & \multicolumn{2}{l|}{ No } & \multicolumn{2}{l|}{ I do not remember } \\
\cline { 2 - 7 } & $\mathbf{N}$ & $\%$ & $\mathbf{N}$ & $\%$ & $\mathbf{N}$ & $\%$ \\
\hline Was fully focused on me and my problem & 150 & 48 & 126 & 40 & 38 & 12 \\
\hline Showed interest in the way I was feeling & 130 & 40.5 & 156 & 50 & 28 & 9.5 \\
\hline Made me feel I that can trust him & 168 & 53.5 & 122 & 39 & 24 & 7.5 \\
\hline Tried to alleviate my worry & 121 & 38.5 & 15 I & 48 & 42 & 13.5 \\
\hline Was being honest with me & 240 & 76.5 & 39 & 12.5 & 35 & 11 \\
\hline
\end{tabular}

Abbreviation: DBN, delivering bad news.

treatment. Patients who decided to cease the medical treatment were also those who three times more often than patients who stayed in contact with their doctor after receiving bad news $(33 \%)$ reported having a feeling that the doctor was downplaying the diagnosis $\left(10 \%\right.$; statistics: $\chi^{2}=28.326$; $d f=2 ; P<0.01)$. Patients who decided to withdraw from the medical treatment under the guidance of the doctor who delivered bad news were also the ones who twice more often $(36 \%)$ reported the usage of incomprehensible medical terms when compared with patients who decided to continue the medical treatment $\left(16 \%\right.$; statistics: $\chi^{2}=17.670 ; d f=2$; $P<0.01)$. We can see clearly that if the negative approach of the doctor discouraged the patients from further contact, so conversely positive approach and supportive behavior of the doctor strengthened the patient-doctor relationship. Patients who decided to continue their medical therapy under the guidance of the doctor who earlier delivered bad news to them almost three times more often confirmed that they were encouraged by the doctor to ask questions (34\% to 13\%; statistics: $\left.\chi^{2}=22.614 ; d f=2 ; P<0.01\right)$ and were twice more often encouraged to individually research and deepen their knowledge about the disease (27\% to $12.5 \%$; statistics: $\left.\chi^{2}=11.077 ; d f=2 ; P<0.01\right)$.

\section{Evaluation of the doctor's approach}

Mutual approach of the interlocutors is an important element that influences the quality of communication. Taking this context as an important point of reference, we aimed to find out the patients' opinions on the doctor's approach during DBN. When asked about the feelings experienced during DBN, $75 \%$ of the patients stated that they felt that doctors were being honest with them while DBN. Over $50 \%$ of the patients stated that they felt they trust the doctor and that during the conversation he/she was fully concentrated on their problem (Table 4).

Similar to analysis outlined above, when evaluating the doctor's approach, we confirmed the presence of a correlation between all mentioned feelings experienced by the patient and their decision to either continue or withdraw from the medical treatment. More than half $(55 \%)$ of the patients who positively evaluated doctor's honesty were also the ones who decided to continue their medical treatment with the same doctor (statistics: $\chi^{2}=17,473 ; d f=2 ; P<0.01$ ). Most of the patients (71\%) who stated that they felt they can trust the doctor decided to continue their medical treatment under the guidance of the same specialist $\left(\chi^{2}=72,237\right.$; $d f=2$; $P<0.01)$. In addition, most of the patients (67\%) who positively evaluated the way the doctor focused on their problem are also those who decided to stay with the same doctor instead of changing him/her to another specialist $\left(\chi^{2}=44,627\right.$; $d f=2 ; P<0.01)$. On the contrary, most of the patients $(66 \%)$ who gave a negative evaluation of the doctor's interest in their case also decided to go to another specialist or to quit the medical treatment altogether $\left(\chi^{2}=44,039 ; d f=2 ; P<0.01\right)$. Analogous situation was found in the group of patients who gave a negative evaluation of the way the doctor tried to counteract their worry and irritation - most of these patients $(70 \%)$ decided to quit their medical treatment under the guidance of the specialist who delivered bad news to them $\left(\chi^{2}=44,192 ; d f=2 ; P<0.01\right)$.

\section{Discussion}

Results that we have obtained with reference to the overall assessment of the DBN process prove that less than a half of patients $(47 \%)$ were pleased with the way the bad news was being delivered to them. These patients have shown also that the doctors have delivered bad news with tact and with proper care. This marker is not very high. However, if we compare these results with the results obtained in the study of patients that were treated by doctors using the SPIKES protocol, we can clearly see that the level of patient's satisfaction is similar (46.2\%). ${ }^{10}$ Also, the marker of negative assessment turned out to be similar. In the study carried out by Greiner and Conklin, 30\% of patients suffered from negative feelings caused by the improperly delivered bad news by doctors using the SPIKES protocol. ${ }^{11}$ Exactly 33\% 
of respondents reported that in the process of DBN the doctors were in no way gentle or careful, which in effect led to negative feelings in the patients. It is worth underlining that in Poland doctors do not use any protocols designed to deliver bad news. Furthermore, within the framework of medical education, students in Poland do not develop communication skills related to the process of DBN. Such courses are not obligatory and are not part of the compulsory education of future physicians. Taking this fact into consideration, we may assume that the results of patients' general satisfaction that we have gathered were relatively high.

In the context of patient's satisfaction and the progress of medical therapy, we can point out two extremely important elements: giving the patient as detailed information as possible about the diagnosis and supplementing this information with an outline of possible consequences of the diagnosis. According to recent studies, most of the patients expect the doctor to provide them with comprehensive and detailed information about the diagnosis and with detailed outline of its possible consequences that would enable them to participate in the process of decision-making as regards their further therapy. ${ }^{12,13}$ On the contrary, involving the patients into decision-making stimulates and increases their engagement in the healing process. ${ }^{14}$ It is worth highlighting that there are certain diseases in the case of which the process of delivering information should foremostly include not just the patient but his or her closest relatives as it may prove to be a condition necessary for the process of taking up therapy. ${ }^{15}$ Our research has shown that in Poland we observe a severe deficit as regards doctors' delivering comprehensive information to patients. Most of the patients reported that they were not provided with information about causes of their disease (40\%), various forms of medical treatment (45\%), health consequences of quitting medical treatment (47\%), and medical recommendations related to changes the patient should implement in their life such as following a prescribed diet and either engaging in or refraining from physical activity (45\%). Exactly $63 \%$ of respondents confirmed that they were in no way encouraged by the doctor to ask any questions or to clarify any problematic issues related to the diagnosis. More than one-fourth of the respondents reported that, while DBN, the doctor was using incomprehensible medical terminology. In this respect, most of the recommendations for doctors are similar in various studies - the patients want bad news to be understandable and devoid of medical terminology. ${ }^{16,17}$ These results support the findings gathered in our earlier studies in which a larger number of patients than doctors expressed their need for partnership and open communication in clinical relationships that will enable them to participate in the process of decision-making as regards their further medical therapy and to fully realize the patient's right to access all the information about their health. ${ }^{8}$

The limited amount of time devoted to communicating the diagnosis is an important factor that strongly influences the dynamics of doctor-patient relationship. Our research has shown that $42 \%$ patients stated that the doctor had too little time for them. In the most recent OECD report, Poland is on the next-to-last place with regard to the amount of time spent on communicating the diagnosis: maximum: Belgium -97.5, OECD32 - 81.3, Poland - 59.6, minimum: Japan-39.0. We should note however that this situation is caused, among other reasons, by the fact that Polish doctors are located on one of the top ranks as regards the number of visits per doctor: Poland - 3,179, OECD32 - 2,295. ${ }^{18}$

If we view this problem from the perspective of communication psychology, we should highlight that the way one interlocutor experiences the other interlocutor's approach and behavior is one of the most important aspects of a dialogical relationship. Therefore, we asked the patients about their feelings related to the doctor's approach and behavior during delivery of bad news. Most of the patients (76.5\%) said that they felt the doctor was being honest with them. According to earlier research, honesty is one of the features that patients claim to be very important for them during DBN. ${ }^{16,19}$ We might thus state that among the study group of doctors that we have analyzed this aspect of doctors' behavior turned out to be positively evaluated by the patients. As for the cognitive and emotional sphere, the patients evaluated the doctors' engagement as poor (Table 4). We are aware of the fact that this aspect is extremely important for the patients and especially important during such visits as DBN. ${ }^{20}$ Furthermore, our research has shown that almost $40 \%$ of patients reported that they felt the doctor to be untrustworthy. In our opinion, this fact might have a direct influence on the effects of clinical treatment. Unfortunately, the doctor-patient relationship still partially reflects the prevalent medical paternalism. One can find a reflection of this situation also in the OECD report in which Polish doctors can be found on last place in the ranking category called - involving patient in decision-making about care and treatment; this results in Poland having a ratio of 47.9 for the average OECD16 - 83.1. ${ }^{18}$

The most significant results of our study are correlations revealed between patients' assessments of the way in which bad news was communicated to them and their decision to continue or stop their medical treatment under the guidance of the specialist who had delivered the bad news to them. 
Patients who gave a negative evaluation of the following elements of DBN process: 1) doctor's behavior while DBN, 2) amount of time the doctor devoted for the patient's visit, 3) lack of doctor's attention, 4) usage of medical terminology, 5) doctor's honesty, (6) doctor's emotional and cognitive support for the patient - more often decide to change their physician in charge and change their medical treatment or decide to quit the treatment altogether.

In the light of the analysis of sociodemographic variables, our results turned out to be analogous to the findings of other researchers. ${ }^{21,22}$ Young people, women, and highly educated patients represent a higher demand for detailed and comprehensive information and they require more attention and emotional support. Regarding the methodology of our research, we have to note however that we used the tool of e-questionnaire. Due to the fact that this research tool and the research technique are in their very nature rather imprecise, we have a limited possibility of generalizing our results for the whole population. This also resulted in the statistical overrepresentation of women who were highly educated and whose average age was 30-40 years. Furthermore, the questionnaire had been accessed only by those people who actively search for information about their disease on the Internet. Taking into account the type of the disease, on the one hand it can be stated that the respondents constitute a relatively diverse group which was our goal. On the other hand, we should note that we have encountered an overrepresentation of oncological patients and patients struggling with neurological diseases. The reason for such a disproportion may be the specificity of the nonprofit sector that participated in spreading information about the questionnaire and reaching the respondents. It is worth mentioning that in order to minimize formal errors at the stage of data preparation, 11 questionnaires were not qualified for analysis due to the lack of detailed information on the type of the respondent's disease or the specialization of the physician providing the diagnosis.

\section{Conclusion}

Our research has shown that patients' opinions and feelings about the way bad news was delivered to them are medically important. Doctors' behavior and the way they deliver bad news are crucial for further outcomes of the therapy treatment. Doctors' behavior and the way they deliver bad news influence patients' decision about quitting or continuing their medical treatment. It also influences patients' decision about changing their physician-in-charge or continuing their medical therapy under the guidance of the doctor who had provided the diagnosis.
Having analyzed particular responses of the patients, we came to a conclusion that in their opinion the doctors are more efficient in terms of delivering technical information (instrumental information) related to their medical knowledge, but they have troubles dealing with expressive communication based on empathy and accurate recognition of others' feelings, emotional states, and emotional needs. We may also note that there is a large difference between the form of DBN and the patients' expectations in this respect.

The results of our research and its conclusions will form the basis for editing the first Polish communication protocol concerning DBN. It is necessary to create such a protocol in order to improve the quality of communication with patients, especially as regards DBN to them.

\section{Informed consent and ethical approval}

Informed consent was obtained from all individual participants included in the study. The research was positively evaluated and approved by the Independent Bioethics Commission for Research at the Medical University of Gdansk.

\section{Acknowledgment}

We would like to express our gratitude to all patients' associations and foundations and to WP Polska ABC Zdrowie for informing patients about free participation in our research.

\section{Disclosure}

The authors report no conflicts of interest in this work.

\section{References}

1. Hulsman RL, Pranger S, Koot S, Fabriek M, Karemaker JM, Smets EM. How stressful is doctor-patient communication? Physiological and psychological stress of medical students in simulated history taking and bad-news consultations. Int J Psychophysiol. 2010;77(1):26-34.

2. Zolnierek KB, Dimatteo MR. Physician communication and patient adherence to treatment: a meta-analysis. Med Care. 2009;47(8):826-834.

3. Golin C, Dimatteo MR, Duan N, Leake B, Gelberg L. Impoverished diabetic patients whose doctors facilitate their participation in medical decision making are more satisfied with their care. J Gen Intern Med. 2002;17(11):857-866.

4. Beck RS, Daughtridge R, Sloane PD. Physician-patient communication in the primary care office: a systematic review. J Am Board Fam Pract. 2002;15(1):25-38.

5. Clark PA. Medical practices' sensitivity to patients' needs. Opportunities and practices for improvement. J Ambul Care Manage. 2003;26(2): $110-123$.

6. Suchman AL, Roter D, Green M, Lipkin M Jr. Physician satisfaction with primary care office visits. Collaborative Study Group of the American Academy on Physician and Patient. Med Care. 1993;31(12): 1083-1092.

7. Ammentorp J, Sabroe S, Kofoed PE, Mainz J. The effect of training in communication skills on medical doctors' and nurses' self-efficacy. A randomized controlled trial. Patient Educ Couns. 2007;66(3): $270-277$. 
8. Sobczak K, Pawłowski L, Pietrzykowska M, Spolak N. Delivering bad news by physicians - Polish reality check. J Med Sci. 2016;85(3): $172-177$.

9. Sobczak K, Leoniuk K, Janaszczyk A, Pietrzykowska M. Patients' expectations as to doctors' behaviors during appointed visits. Health Commun. 2017;32(4):517-519.

10. Seifart C, Hofmann M, Bär T, Riera Knorrenschild J, Seifart U, Rief W. Breaking bad news-what patients want and what they get: evaluating the SPIKES protocol in Germany. Ann Oncol. 2014;25(3):707-711.

11. Greiner AL, Conklin J. Breaking bad news to a pregnant woman with a fetal abnormality on ultrasound. Obstet Gynecol Surv. 2015;70(1): 39-44.

12. Brown VA, Parker PA, Furber L, Thomas AL. Patient preferences for the delivery of bad news - the experience of a UK Cancer Centre. Eur J Cancer Care (Engl). 2011;20(1):56-61.

13. Davison BJ, Parker PA, Goldenberg SL. Patients' preferences for communicating a prostate cancer diagnosis and participating in medical decision-making. BJU Int. 2004;93(1):47-51.

14. Edwards A, Elwyn G. Inside the black box of shared decision making: distinguishing between the process of involvement and who makes the decision. Health Expect. 2006;9(4):307-320.
15. Outram S, Harris G, Kelly B, et al. "We didn't have a clue": family caregivers' experiences of the communication of a diagnosis of schizophrenia. Int J Soc Psychiatry. 2015;61(1):10-16.

16. Kim MK, Alvi A. Breaking the bad news of cancer: the patient's perspective. Laryngoscope. 1999;109(7 Pt 1):1064-1067.

17. Randall TC, Wearn AM. Receiving bad news: patients with haematological cancer reflect upon their experience. Palliat Med. 2005;19(8): 594-601.

18. OECD. Health at a Glance 2017. OECD Indicators. Paris: OECD; 2017:1-220.

19. Ishaque S, Saleem T, Khawaja FB, Qidwai W. Breaking bad news: exploring patient's perspective and expectations. J Pak Med Assoc. 2010; 60(5):407-411.

20. Martins RG, Carvalho IP. Breaking bad news: patients' preferences and health locus of control. Patient Educ Couns. 2013;92(1):67-73.

21. Fujimori M, Uchitomi Y. Preferences of cancer patients regarding communication of bad news: a systematic literature review. Jpn J Clin Oncol. 2009;39(4):201-216.

22. Parker PA, Baile WF, de Moor C, Lenzi R, Kudelka AP, Cohen L. Breaking bad news about cancer: patients' preferences for communication. J Clin Oncol. 2001;19(7):2049-2056.

\section{Publish your work in this journal}

Patient Preference and Adherence is an international, peer-reviewed, open access journal that focuses on the growing importance of patient preference and adherence throughout the therapeutic continuum. Patient satisfaction, acceptability, quality of life, compliance, persistence and their role in developing new therapeutic modalities and compounds to optimize clinical outcomes for existing disease states are major areas of interest for the journal. This journal has been accepted for indexing on PubMed Central. The manuscript management system is completely online and includes a very quick and fair peer-review system, which is all easy to use. Visit http://www dovepress.com/testimonials.php to read real quotes from published authors. 